\title{
Review: long term lithium treatment lowers suicide risk in major affective disorder
}

Tondo L, Hennen J, Baldessarini RJ, et al. Lower suicide risk with long-term lithium treatment in major affective illness: a meta-analysis. Acta Psychiatr Scand 2001 Sep;104:163-72.

\section{QUESTION: In patients with a major affective disorder, is long term lithium treatment associated with lower suicide risk?}

\section{Data sources}

Studies were identified by searching Current Contents, Medline, PsycLIT, and PubMed from the 1960s. Also, pertinent publications (since 1949) were searched and experts were contacted.

\section{Study selection}

Studies were selected if they examined lithium treatment in patients with bipolar manic depressive disorder, major affective disorder (including recurrent major depression), or schizoaffective disorder and included data for estimating suicide rates.

\section{Data extraction}

Data were extracted on patient diagnoses, study design, number of completed suicides, total number of patients at risk of suicide, at risk exposure times, and study quality. Study quality was assessed using a quality scoring scale (maximum score 7, which referred to the highest quality score) and expressed as a percentage of the maximum.

\section{Main results}

22 studies (5647 patients, 33473 patient years of risk, mean quality rating $47,3 \%$ randomised trials), were identified that included data on suicide risk data during maintenance lithium treatment (weighted mean treatment duration 6.02 y). Of the 22 studies, 13 also provided suicide risk data on 1439 patients who were not receiving lithium treatment (mean duration of observation 5.03 y). Based on a random effects model (22 studies), the overall weighted suicide rate during lithium treatment was $0.16 \%$ year (95\% CI $0.13 \%$ to $0.20 \% / y$ ) and the weighted suicide rate when off lithium treatment was $0.88 \% /$ year (CI $0.63 \%$ to $1.12 \% / y$ ). Suicide rates with and without lithium treatment were reported in 12 studies; all of these studies showed higher suicide risks when off than when on lithium treatment (weighted risk ratio 8.85 , CI 4.14 to $19.1, \mathrm{p}<0.0001$; random effects model). Similar results were seen when the analysis was confined to the 3 randomised trials \{weighted risk ratio 9.1, CI 1.2 to 100$\}$.*

\section{Conclusion}

In patients with a major affective disorder, long term lithium treatment lowers suicide risk.

*Calculated from data in article.

\section{COMMENTARY}

The question of whether prophylactic lithium reduces the risk of suicide in mood disorders is clinically important, both because of the high rate of suicide in mood disorder and the lack of evidence that other treatments (such as antidepressants) affect suicide rates. All research in suicide prevention faces the common challenges of the rarity of suicide (even in high risk groups) and the ethical constraints of clinical trials on suicide. Good evidence on this question has been difficult to obtain.

This meta-analysis by Tondo et al involves a careful search for all relevant clinical trials. It concludes that patients with mood disorder who use lithium have lower rates of suicide than those who do not use lithium. This finding is in agreement with other recent reviews. ${ }^{12}$

The major limitation of this meta-analysis is that it is not confined to randomised controlled trials - in fact, several of the included studies did not use parallel control groups at all. This raises the issue of the comparability of patients who did and did not take lithium. For example, some of the studies used control groups comprising patients who had dropped out of lithium treatment. Such patients are likely to differ substantially from patients who continued to take lithium; patients who were able to tolerate the discipline of lithium treatment may have been at lower inherent risk of suicide than those unable or unwilling to comply.

Thus, although the size of the antisuicide effect found in this meta-analysis is striking, and was supported by the few randomised trials available, it remains uncertain as to what extent the reduction in risk with lithium is a treatment effect, rather than a between patient difference. If lithium does exert a true treatment effect, the mechanism of action is unclear. It could be either a direct antisuicidal effect or an effect secondary to prevention of relapse, particularly into depression. Only the large scale randomised controlled trials in non-selected groups of patients comparing lithium with another effective maintenance treatment that uses suicide (or a suitable proxy) as an outcome measure will give a clearer answer to this question.

Sally Burgess, MRCPsych Shropshire's Community and Mental Health NHS Trust Shrewsbury, UK

1 Crundwell JK. Lithium and its potential benefit in reducing increased mortality rates due to suicide. Lithium 1994;5:193204.

2 Davis JM, Janicak PG, Hogan DM. Mood stabilizers in the prevention of recurrent affective disorders; a meta-analysis. Acta Psychiatr Scand 1999:100:406-17. 